\title{
Evaluación del uso de almidón de papa como aditivo para lodos de perforación
}

\author{
Juan Carlos Santivañez Huarcaya ${ }^{1 *}$, Walter Erick Cano Tocas ${ }^{2}$, Daniela Ordoñez Ruiz ${ }^{3}$, Andrea del Pilar Bohórquez Araque ${ }^{4}$ \\ ${ }^{1}$ Bachiller en Ingeniería Química del Gas Natural y Energía. Universidad Nacional del Centro del Perú (UNCP). \\ Av. Mariscal Castilla N³909 El Tambo, Huancayo. Perú. \\ ${ }^{2}$ Bachiller en Ingeniería Química del Gas Natural y Energía. Universidad Nacional del Centro del Perú (UNCP). \\ Av. Mariscal Castilla N³909 El Tambo, Huancayo. Perú. \\ ${ }^{3}$ Universidad Industrial de Santander (UIS), Carrera 27 calle 9. Bucaramanga, Colombia. \\ ${ }^{4}$ Universidad Industrial de Santander (UIS). Carrera 27 calle 9. Bucaramanga, Colombia. \\ E-mail: jc.santivanez.h@uncp.edu.pe
}

\begin{abstract}
Resumen
El uso de almidones en la formulación de fluidos de perforación se viene dando desde 1930, principalmente como controladores de filtrado y extensores de viscosidad. Los almidones de maíz y de papa son los que más vienen usándose, sin embargo, se sigue en la búsqueda de aditivos naturales que puedan reemplazar a los comerciales. El almidón de papa es un polímero natural, cuya relación amilosa/amilopectina y el contenido de fósforo en forma de éster de fosfato, lo hace superior frente a productos como el maíz, la yuca y el plátano. La posibilidad del uso de almidón de papa, producido en Perú, como aditivo para lodos de perforación, se contempla debido a que el país es considerado uno de los 10 mayores productores de este tubérculo a nivel mundial. Sin embargo, hay una ausente industrialización del mismo lo que representa una oportunidad de desarrollo de la papa y el almidón de papa en el país.
\end{abstract}

Este trabajo evaluó el efecto del almidón de papa y $\mathrm{NaOH}$ en el filtrado API de un fluido de perforación base agua con un diseño factorial mixto. De acuerdo a los resultados en esta investigación se encontró que el almidón de papa muestra mejor propiedad de control de filtrado puesto que con solo $0.5 \mathrm{lb} / \mathrm{bbl}$ se obtuvo una disminución de pérdida de filtrado respecto a un lodo Bentonitico base agua con PAC-L. Así mismo, se comprobó que se puede reemplazar el PAC L por almidón de papa para utilizarlo como controlador de filtrado en un lodo base agua, además de mejorar la viscosidad y las propiedades reológicas del lodo, contribuyendo así con la sostenibilidad del medio ambiente y generando un impacto positivo en la industria en la reducción de costos operacionales y oportunidad de desarrollo industrial de la papa y sus derivados en el Perú.

Palabras claves: Almidón; Papa; PAC-L; Filtrado; Viscosidad.

\section{Evaluation of the Use of Potato Starch as an Additive for Drilling Mud}

\begin{abstract}
The use of starches in the formulation of drilling fluids has been taking place since the 1930s, mainly as filter controllers and viscosity extenders. Corn and potato starches are the most used; moreover, the search for natural additives that could replace the commercial ones continues. Potato starch is a natural polymer whose amylose/amylopectin ratio and phosphorus content in the form of phosphate ester makes it superior to products such as corn, cassava, and plantain. The possibility of using potato starch, produced in Peru, as a drilling mud additive is contemplated as the country is considered one of the top 10 producers of this tuber worldwide. However, there is an absent industrialization of it, which represents an opportunity for potato and potato starch development in the country.
\end{abstract}

This research evaluated the effect of potato starch and $\mathrm{NaOH}$ in the API filtrate of a water-based drilling fluid with mixed factorial design. According to the results obtained in this research, it was found that potato starch shows a better filter control property since with only $0.5 \mathrm{lb} / \mathrm{bbl}$ a decrease in filter loss was obtained with respect to a water-based Bentonitic sludge

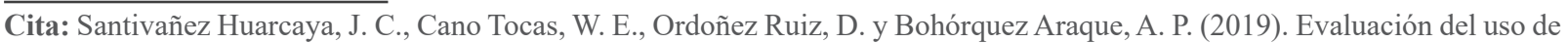
almidón de papa como aditivo para lodos de perforación. Revista Fuentes: El reventón energético, 17(1), 19-28. 
with PAC-L. Likewise, it was proved that the PAC L can be replaced by the potato starch so that it can be used as a filter controller in a water-based mud, and to improve the rheological and viscosity properties of the same; contributing with this to the environmental sustainability, and generating a positive impact in the industry in the reduction of operational costs, and the industrial development of potato and its derivatives in Peru.

Key words: starch, potato, PAC-L, filtration, viscosity.

\section{Introducción}

Una de las principales actividades de la extracción de hidrocarburos es la perforación de pozos, la cual ha ido evolucionando a pasos agigantados en el desarrollo tecnológico de los materiales utilizados en esta operación. Los almidones de maíz y papa se han venido usando desde 1930 en esta actividad valiéndose de su facilidad para la degradación por tratarse de un polímero natural.

El almidón está compuesto por dos polímeros: amilosa y amilopectina. En presencia de agua y calor las moléculas de amilosa se agrupan para formar un gel que aumenta la viscosidad del sistema y es lo suficientemente fuerte para taponar microfacturas presentes en las paredes del pozo.

El almidón de papa, a diferencia de otros almidones presenta una relación amilosa/amilopectina de 20/80 respectivamente, además de su contenido de fósforo que contribuye a su alta capacidad de retención de agua, viscosidad, claridad y la estabilidad a la congelacióndescongelación.

Bajo contenido de monoéster de fosfato se encuentra en el almidón de cereales. Los fosfolípidos presentes en el almidón (por ejemplo, de trigo, arroz y maíz) tienen una tendencia a formar complejos con la amilosa y las cadenas ramificadas largas de amilopectina, lo que limita la hinchazón del gránulo de almidón, y se obtienen geles opacos y de baja viscosidad. (Kaur, Singh, Ezekiel, \& Guraya, 2007) (Sandhu \& Singh, 2007).

La papa tiene una amplia gama de usos: como alimento básico (para consumo fresco y en forma procesada), en cultivos comerciales para alimentación animal y como materia prima con fines industriales (MINAGRI, 2014). Perú lidera la producción de papa en Latinoamérica, siendo esta de 4,6 millones de toneladas al año y el consumo aproximado de este producto es de $80 \mathrm{~kg}$ por persona al año. (Direccion General de Seguimiento y Evaluación de Politicas - DGESEP, 2017)

Además de ser de los 10 mayores productores mundiales de este tubérculo, superando así el consumo interno, caso reciente en la campaña de cosecha del 2018 en la que se tuvo una sobreproducción y posterior caída de precios y pérdida de producto por falta de industrialización.
El uso de estos remanentes de sobreproducción para la industrialización de almidón de papa como aditivo de lodo de perforación, no afecta la sostenibilidad del uso del producto la cadena alimenticia nacional.

\section{Marco teórico}

\section{Componentes de un lodo convencional}

Los principales productos químicos que se le añaden a un fluido de perforación son los viscosificantes, densificantes, controladores de filtrado y controladores de $\mathrm{pH}$ (Ayala, Benítez, \& Valencia, 2017); (Portilla, Suárez, \& Corzo, 2012); (Campana \& Tapia, 2017)). Siendo estos productos los que mantienen las propiedades reológicas del fluido de perforación. (AMOCO Production Company, 1998)

\section{Bentonita (Montmorillonita de Sodio)}

Principalmente compuesta por montmorillonita de sodio (Medina, Martínez, León , \& Boada, 2013). Esta clase de arcilla puede ofrecer un muy buen aumento de la viscosidad del fluido y control de pérdidas de fluido para lodos base agua debido a la alta tasa de absorción de agua que presentan sus gránulos. (Bautista Cuadros \& Flórez Barreto, 2011)

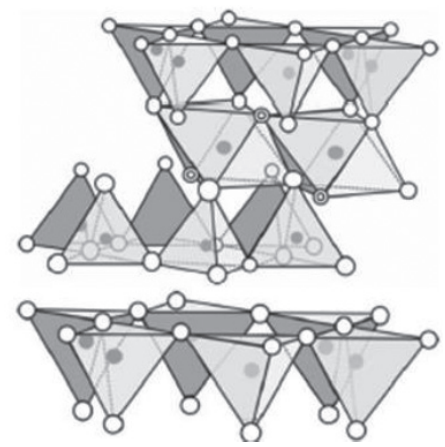

Figura $\mathrm{N}^{\circ}$ 1. Estructura de la Bentonita

Fuente: (PDVSA CIED Petróleos de Venezuela, S.A. Centro Imternacional de Educación y Desarrollo, 2002)

\section{Almidón de papa}

Las sustancias que se encuentran comúnmente en los gránulos de almidón son amilopectina, amilosa, moléculas intermedias entre la amilosa y la amilopectina, lípidos (incluyendo fosfolípidos y ácidos grasos libres), monoéster de fosfato y proteínas/enzimas. 
Los polisacáridos amilosa y la amilopectina son los componentes más abundantes. El contenido de amilosa del almidón de papa varía del 23 al 31 por ciento para los genotipos normales de papa, ya que, con respecto a este, a través de la modificación genética se ha producido almidón de alta amilopectina (Bemiller \& Whistler, 2009).
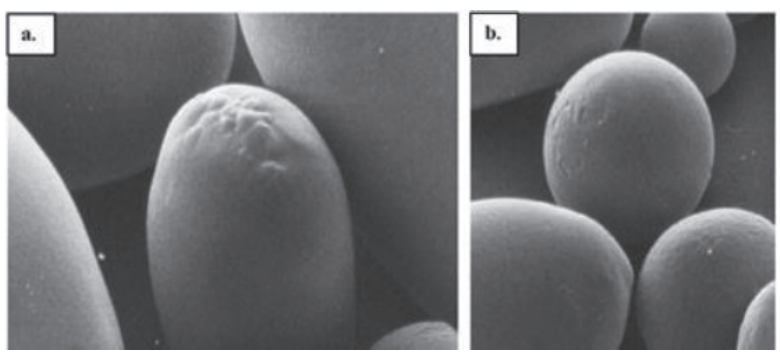

Figura $\mathbf{N}^{\circ}$ 2. Barrido de micrografías electrónicas (SEM) (a) la presencia de protuberancias en algunos gránulos de almidón de papa y (b) la fragmentación de las superficie en algunos gránulos de almidón de papa

Fuente: (Peña Carrasco, 2017)

El almidón de papa es usado prácticamente para cualquier tipo de sistema lodo base agua dulce hasta los sistemas saturados de sal y de calcio. Por esto es un aditivo eficaz de control de filtración para perforar las secciones de evaporita (sal) y lutita hidratable. También es muy eficaz para estabilizar la filtración y la reología de las salmueras de alta salinidad usadas en las operaciones de rehabilitación (Ortiz-Vargas et al, 2018). Estos almidones son estables hasta temperaturas aproximadas de $240{ }^{\circ} \mathrm{F}$, después de esta temperatura sufren una degradación. (MI -SWACO, 1998).

El proceso de extracción de almidón de tubérculos de papa es relativamente sencillo debido a la estructura del tejido y su bajo contenido en proteínas y grasas. Los principales pasos de extracción incluyen lavado del tubérculo, desintegración, y centrifugación. Por ende, se requieren pocas unidades de producción para este proceso.

\section{PAC-L}

El aditivo de control de filtración PAC-L ${ }^{\text {TM }}$ se usa en la mayoría de los fluidos de perforación a base de agua, incluyendo agua dulce, agua de mar y salmuera hasta $300^{\circ} \mathrm{F}\left(149^{\circ} \mathrm{C}\right)$ (Ayala, Benítez, \& Valencia, 2017). Además, es un polímero natural modificado que está diseñado para ser usado en la mayoría de los sistemas base agua, incluyendo los lodos de agua dulce, lodos de agua salada, lodos salados y lodos de bajo contenido de sólidos (Loaiza, Ayala, Torres, \& Ayala, 2018).
Ésta es una celulosa polianiónica de alto peso molecular que es similar a la CMC, pero tiene un grado de substitución más alto. El PAC no está sujeto a la degradación por actividad bacteriana y es eficaz cuando el pH es alcalino. El PAC está sujeto a la degradación térmica a temperaturas que exceden $275^{\circ} \mathrm{F}$, además de un impacto muy bajo en la reología de fluidos, no es tóxico y no requiere un biocida. (Halliburton Baroid, 2017).

\section{$\mathrm{NaOH}$}

El nombre común del hidróxido de sodio $\mathrm{NaOH}$. La soda cáustica se utiliza en la mayoría de los lodos a base de agua para aumentar y mantener el pH y la alcalinidad (Ayala , Gómez, \& León, 2011). Es un material peligroso de manipular porque es muy cáustico y emite calor cuando se disuelve en agua (Schlumberger Limited, 2018).

\section{Metodología}

Para esta investigación se usó almidón de papa, a partir de chuño variedad única, con características adecuadas para las pruebas experimentales como aditivo en fluidos de perforación base agua. Para evaluar la factibilidad del uso del almidón de papa como aditivo de lodos de perforación base agua y observar la relación con $\mathrm{NaOH}$, se programaron pruebas con base en la norma API 13B-1 de $\mathrm{pH}$, densidad, viscosidad y fuerza de gel, filtración y contenido de sólidos, agua y aceite del lodo para establecer la incidencia del aditivo sobre las demás propiedades.

Se estableció la relación entre el almidón de papa y $\mathrm{NaOH}$, además de su influencia en el diseño de un fluido base agua con los agentes antes mencionados. (American Petroleum Insititute, 2017). Para desarrollar las pruebas mencionadas se usó un diseño experimental factorial mixto (Montgomery, 2004).

\section{Obtención del almidón de papa}

En la papa, los gránulos de almidón se encuentran libres en el interior de las vacuolas celulares, de modo que su aislamiento es relativamente sencillo. El material vegetal se tritura, los gránulos de almidón se arrastran en corriente de agua, se sedimentan a partir de la suspensión (leche de almidón) y se desecan (Peña Carrasco, 2017).

Los principales pasos de extracción incluyen lavado del tubérculo, desintegración, y centrifugación. El remojo se lleva a cabo en una solución de bisulfito 
de sodio a $\mathrm{pH}$ controlado para evitar el pardeamiento del tubérculo. La desintegración y la centrifugación se utilizan para separar el almidón de otros componentes. Los gránulos de almidón se distribuyen de forma desigual en las paredes celulares de los tubérculos. Pueden ser liberados de tubérculo por la ruptura de las paredes celulares. Esto se hace durante la desintegración de tubérculos por un tambor cilíndrico que contiene hojas de sierra rotativas en su circunferencia o un extractor de jugo para la extracción en una pequeña escala. El almidón puede purificarse por lavado, sedimentación y centrifugación (Cui, 2005).

Para las pruebas se utilizó almidón de papa (chuño variedad única), el cual se extrajo por el procedimiento descrito por (Soto Izarra \& Yantas Huaynate, pág. 98). A este almidón se le realizó la prueba de Yodometría en la que resultó superior el contenido de almidón con respecto a otros almidones comerciales de Perú. Este almidón presenta un color claro-blanco.

\section{Prueba de Rendimiento}

El objetivo de comparar el rendimiento del almidón de papa con otros aditivos de lodos comúnmente utilizados en la industria petrolera se realizó para determinar si el almidón de papa con una cantidad menor a $99.85 \mathrm{~kg} /$ $\mathrm{m}^{3}(35 \mathrm{lb} / \mathrm{bbl})$ alcanza la viscosidad de $15 \mathrm{cP}$.

Para esto se agregaron pequeñas cantidades de almidón de papa a la bentonita (viscosificante principal) para evaluar su efectividad como viscosificante y extendedor de las propiedades viscosificantes.

Se agregaron cantidades constantes de almidón de papa, se agitó por $3 \mathrm{~min}$ a temperatura ambiente y se midió la viscosidad aparente (Rodriguez Bermudez, 2007), luego se continuó agregando cantidad del aditivo hasta alcanzar una viscosidad aparente superior a $15 \mathrm{cP}$.

Finalmente se construyó una curva de concentración del viscosificante versus la viscosidad aparente. Para esto se utilizó un viscosímetro rotacional 35-A (Fann Instrument Company) de acuerdo a las especificaciones del fabricante, el error del equipo corresponde a $+0.5 \mathrm{Cp}$.

\section{Prueba de Densidad}

Se midió la densidad del fluido tratado con almidón de papa para comparar valores con aditivos comerciales y determinar su compatibilidad de uso. Se utilizó la balanza de lodos a presión atmosférica. (Baker Hughes, 2006).

\section{Prueba de $\mathrm{pH}$}

Esta prueba es el principal indicador de la degradación del producto. Se usaron tiras indicadoras de $\mathrm{pH}$; de acuerdo a la especificación de la fábrica el error es \pm $0,05 \mathrm{pH}$. La medición del pH se realizó a todos los lodos, buscando establecer la variabilidad de este a las diferentes concentraciones de almidón de papa y $\mathrm{NaOH}$. (Baker Hughes, 2006) (American Petroleum Insititute, 2017).

\section{Prueba de Viscosidad y Fuerza de gel}

Se realizaron pruebas de viscosidad plástica $(\mathrm{Vp})$ en $\mathrm{Cp}$, el punto de cedencia o yield point (Yp) en lbf/100pies2 $\left(0,0489 \mathrm{~kg} / \mathrm{m}^{3}\right)$, la viscosidad aparente (Va) medida en $\mathrm{Cp}$, el punto de gel (Gel) en lbf/100pies2 a 10 s y $10 \mathrm{~min}$. Estas pruebas se realizaron en un viscosímetro rotacional de velocidad variable (1 a 600rpm) de referencia OFITE 900. (Baker Hughes, 2006) (American Petroleum Insititute, 2017)

\section{Resultados y discusión}

\section{Prueba de Rendimiento}

En la Figura $\mathrm{N}^{\circ} 3$ se observa el comportamiento de la viscosidad aparente de 3 sistemas de fluidos con respecto a la concentración de cada agente potenciador de la viscosidad y en la Tabla $\mathrm{N}^{\circ} 1$ se presenta el rendimiento de cada uno. Se observa que la concentración de aditivo más baja (concentración a $15 \mathrm{cP}$ ) corresponde a la Bentonita y que el peor rendimiento corresponde al de Almidón de Papa.

El rendimiento de la Bentonita nacional cuya concentración para lograr el rendimiento alcanza los $79.88 \mathrm{~kg} / \mathrm{m} 3$ (28 lb/barril). Por último, se observa el almidón de papa el cual necesita una concentración de más de $40 \mathrm{lb} / \mathrm{bbl}$ de producto que los aditivos anteriormente analizados.

Tabla No 1. Prueba de Rendimiento.

\begin{tabular}{|l|l|}
\hline \multicolumn{1}{|c|}{ Producto } & \multicolumn{1}{|c|}{ Rendimiento (lb/barril) } \\
\hline Bentonita Nacional & 28 \\
\hline Almidón de Papa & Mayor a 40 \\
\hline $\begin{array}{l}\text { Bentonita Nacional + Almidón de } \\
\text { Papa }\end{array}$ & $\begin{array}{l}65 \text { (32.5 de bentonita }+32.5 \text { de } \\
\text { almidón) }\end{array}$ \\
\hline
\end{tabular}

\section{Prueba de Densidad y $\mathbf{p H}$}

La densidad de las muestras estuvo dentro de los parámetros de operación de un lodo base agua y el pH dentro de los rangos establecidos de 10 y 11(ver Figura $\mathrm{N}^{\circ} 4$ y Figura $\mathrm{N}^{\circ} 5$ ), siendo valores adecuados para un lodo que sufrirá cambios de $\mathrm{pH}$ por las formaciones que atravesará y previniendo la corrosión de los equipos dentro del pozo. 


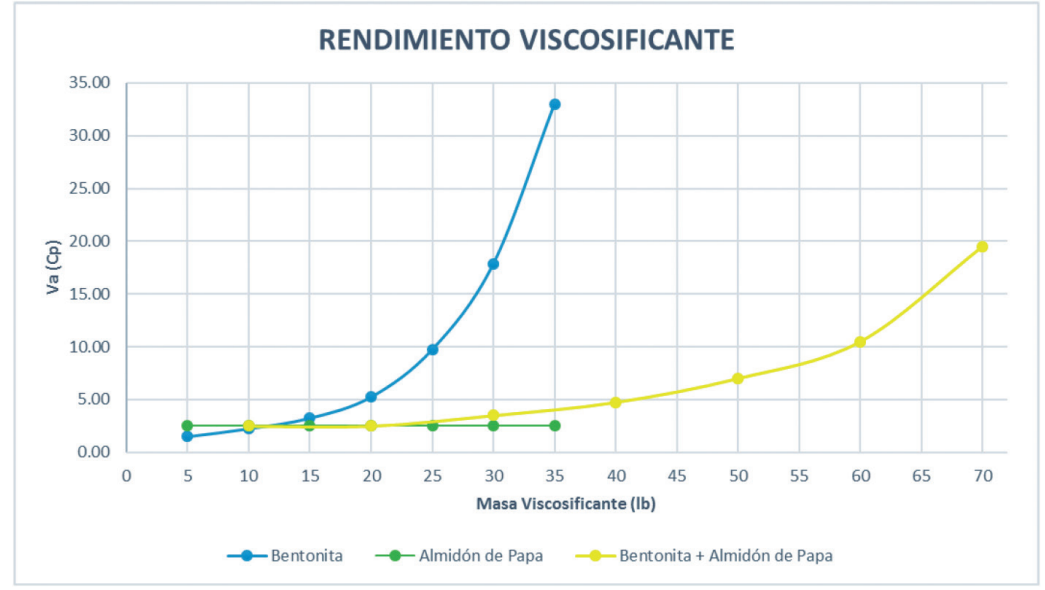

Figura $N^{\circ}$ 3. Rendimiento Viscosificante

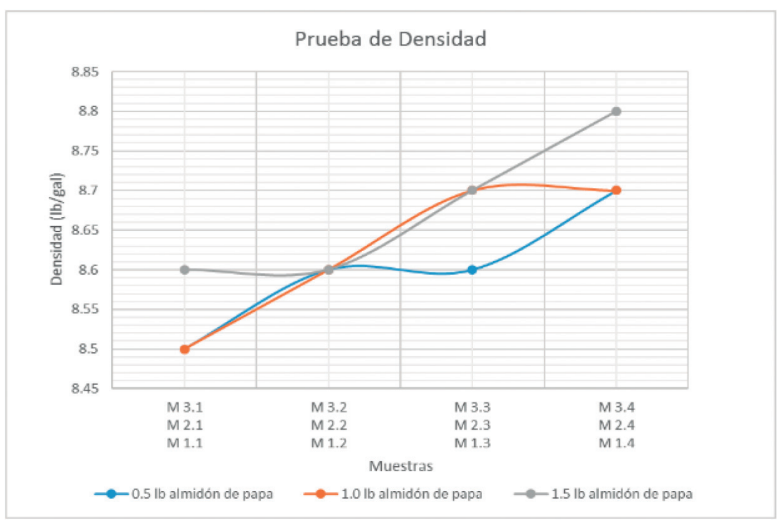

Figura $N^{\circ}$ 4. Prueba de Densidad

\section{Prueba de Viscosidad y Fuerza de gel}

En las pruebas de reología notamos un incremento en la viscosidad y en el punto de gel, teniendo buenos resultados de viscosidad en las concentraciones de $\mathrm{NaOH}$ de entre 0.27 y $0.37 \mathrm{lb} / \mathrm{bbl}$ y de todas las concentraciones de Almidón de Papa experimentadas.

El $\mathrm{NaOH}$ incrementa significativamente la viscosidad del fluido, esto debido a su propiedad exotérmica que posiblemente activó térmicamente al Almidón de Papa, por lo que se nota el incremento de la viscosidad en las muestras, mientras que a mayor concentración de $\mathrm{NaOH}$ disminuye el valor de viscosidad al igual que el Yield Point. Esta prueba indica que el almidón de papa cumple con las propiedades de agente extensor de la Viscosidad de la Bentonita y con el $\mathrm{NaOH}$ el almidón se activa térmicamente, con lo que se incrementa el valor de viscosidad.

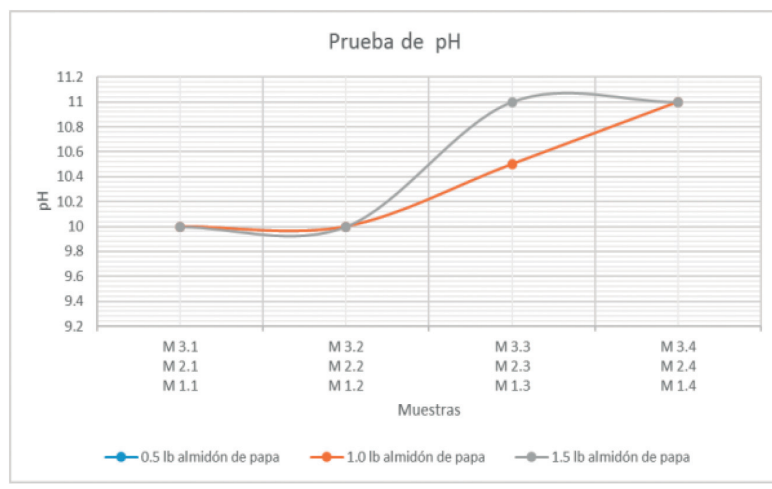

Figura $\mathbf{N}^{\circ}$ 5. Prueba de $\mathrm{pH}$

\section{Prueba de Filtrado}

La prueba de filtrado API se realizó a temperatura ambiente y a una presión de 100psi $(689,4 \mathrm{kPa})$, obteniéndose una torta del lodo por la acumulación de sólidos mediante el proceso de filtración; una buena torta debe tener entre $1 / 32$ in $(0,079 \mathrm{~cm})$ y $2 / 32$ in $(0,015 \mathrm{~cm})$ de espesor, ser plástica y consistente.

En la presente investigación se realizaron pruebas a 12 lodos base agua bentonita con un $\mathrm{pH}$ de entre 10 - 11 y densidad de entre $8.5 \mathrm{lb} / \mathrm{gal}(1013 \mathrm{~g} / \mathrm{l})$ y $8.8 \mathrm{lb} /$ gal $(1048 \mathrm{~g} / \mathrm{l})$, donde se evaluó el comportamiento del almidón de Papa y $\mathrm{NaOH}$ en el filtrado. La mayor parte de controladores de filtrado utilizados en fluidos de perforación base agua logran su efectividad en una concentración entre 0,5 y 8lb/bbl. (MI -SWACO, 1998).

Las concentraciones utilizadas para el almidón de papa variaron entre $0,5-1.5 \mathrm{lb} / \mathrm{bbl}(0,001429-0,00428 \mathrm{~g} /$ $\mathrm{cm}^{3}$ ); se realizó un seguimiento del comportamiento del 
volumen de filtrado del lodo (ml) a 30 min. (American Petroleum Insititute, 2017).

En la Figura $\mathrm{N}^{\circ}$ 9, se observa el comportamiento de las pérdidas de filtrado a 30min (prueba API) respecto a la concentración de PAC L y almidón de Papa. Comparando a una concentración de $0.5 \mathrm{lb} / \mathrm{bbl}\left(0,0014 \mathrm{~g} / \mathrm{cm}^{3}\right)$, la pérdida de filtrado es menor en cerca del $12 \%$ para el almidón de papa frente al PAC L.
Al observar los resultados se encuentra que la concentración de $0.5 \mathrm{lb} / \mathrm{bbl}$ de almidón de papa obtiene un mejor control de filtrado a comparación del PAC-L en $6 \mathrm{ml}$, lo cual corrobora que las pérdidas de filtrado usando Almidón de Papa disminuyen, lo que lo hace un aditivo que reemplaza de forma óptima al Control de Filtrado Comercial.

Tabla $\mathbf{N}^{\circ}$ 2. Resultados de las pruebas de Densidad, $\mathrm{pH}$, Viscosidad y Esfuerzo de gel

\begin{tabular}{|c|c|c|c|c|c|c|c|c|c|c|}
\hline \multicolumn{2}{|c|}{$\begin{array}{c}\text { Concentración } \\
(\mathbf{l b} / \mathbf{b} b l)\end{array}$} & \multirow[b]{2}{*}{ Densidad } & \multirow[b]{2}{*}{ pH } & \multicolumn{5}{|c|}{ Viscosidad } & \multicolumn{2}{|c|}{$\begin{array}{c}\text { Esfuerzo gel } \\
\left(\text { lbf } / 100 f^{2}{ }^{2}\right)\end{array}$} \\
\hline $\begin{array}{c}\text { Almidón de } \\
\text { papa }\end{array}$ & $\mathrm{NaOH}$ & & & $\theta 600$ * & $\theta 300$ ** & $V p(c P)$ & $\mathrm{Va}(\mathrm{cP})$ & $\begin{array}{c}\text { Yp } \\
\left(\text { Ipf/100 pies }{ }^{2}\right)\end{array}$ & $10 \mathrm{~s}$ & $10 \mathrm{~min}$ \\
\hline \multirow{4}{*}{0.5} & 0.17 & 8.5 & 10 & 155 & 123 & 32 & 77.5 & 91 & 31 & 50 \\
\hline & 0.27 & 8.6 & 10 & 179 & 150 & 29 & 89.5 & 121 & 38 & 61 \\
\hline & 0.37 & 8.6 & 10.5 & 174 & 138 & 36 & 87 & 102 & 32 & 48 \\
\hline & 0.50 & 8.7 & 11 & 63 & 52 & 11 & 31.5 & 41 & 5 & 30 \\
\hline \multirow{4}{*}{1.0} & 0.17 & 8.5 & 10 & 195 & 154 & 41 & 97.5 & 113 & 31 & 50 \\
\hline & 0.27 & 8.6 & 10 & 188 & 150 & 38 & 94 & 112 & 34 & 51 \\
\hline & 0.37 & 8.7 & 11 & 182 & 115 & 67 & 91 & 48 & 28 & 43 \\
\hline & 0.50 & 8.7 & 11 & 47 & 39 & 8 & 23.5 & 31 & 4 & 28 \\
\hline \multirow{4}{*}{1.5} & 0.17 & 8.6 & 10 & 100 & 75 & 25 & 50 & 50 & 30 & 53 \\
\hline & 0.27 & 8.6 & 10.5 & 181 & 144 & 37 & 90.5 & 107 & 32 & 43 \\
\hline & 0.37 & 8.7 & 11 & 177 & 118 & 59 & 88.5 & 59 & 23 & 25 \\
\hline & 0.50 & 8.8 & 11 & 38 & 23 & 15 & 19 & 8 & 2 & 24 \\
\hline
\end{tabular}

* lectura del viscosímetro a $600 \mathrm{rpm}$

** lectura del viscosímetro a $300 \mathrm{rpm}$

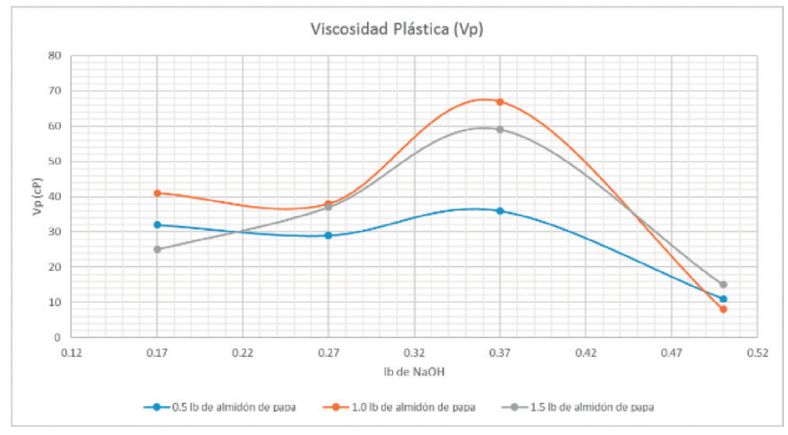

Figura $N^{\circ}$ 6. Viscosidad Plástica.



Figura $\mathbf{N}^{\circ}$ 7. Viscosidad Aparente.

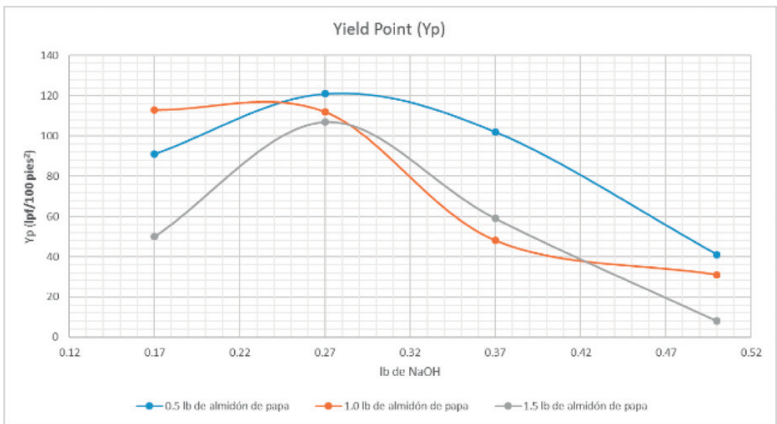

Figura $N^{\circ} 8$. Yield Point.

\section{Prueba de Contenido de Sólidos, agua y aceite}

Para determinar la cantidad de líquidos y sólidos en un fluido de perforación se requiere el uso de una retorta. Equipo de destilación que vaporiza la muestra de lodo y condensa la cantidad de líquido presente en la muestra. Se lee el porcentaje de agua, aceite y solidos directamente en la probeta graduada, una o dos gotas de solución atomizada ayudan a definir el contacto aceiteagua después de leer el porcentaje de sólidos. 
Tabla $\mathbf{N}^{\circ}$ 3. Resultados de la prueba de Filtrado.

\begin{tabular}{|c|c|c|c|c|c|c|c|c|c|}
\hline \multicolumn{2}{|c|}{ FILTRADO } & \multirow{3}{*}{ Consistencia } & \multirow{3}{*}{ Elasticidad } & \multirow{3}{*}{ Espesor } & \multicolumn{5}{|c|}{ Filtrado (ml) } \\
\hline \multirow{2}{*}{$\begin{array}{l}\text { Almidón de papa } \\
\text { (lb) }\end{array}$} & \multirow{2}{*}{$\begin{array}{c}\mathrm{NaOH} \\
\text { (lb) }\end{array}$} & & & & 7.5 & 15 & 20 & 25 & 30 \\
\hline & & & & & $\min$ & $\min$ & $\min$ & $\min$ & $\min$ \\
\hline \multirow{4}{*}{0.5} & 0.17 & $\mathrm{Si}$ & $\mathrm{Si}$ & $>1 / 32$ “ & 20.0 & 23.0 & 24.5 & 26.0 & 27.5 \\
\hline & 0.27 & $\mathrm{Si}$ & $\mathrm{Si}$ & $>1 / 32$ “ & 8.0 & 11.0 & 11.5 & 13.0 & 14.5 \\
\hline & 0.37 & $\mathrm{Si}$ & $\mathrm{Si}$ & $>1 / 32$ “ & 14.0 & 17.0 & 18.5 & 20.0 & 21.5 \\
\hline & 0.50 & $\mathrm{Si}$ & $\mathrm{Si}$ & $>1 / 32$ “ & 7.0 & 9.0 & 10.5 & 11.0 & 12.0 \\
\hline \multirow{4}{*}{1.0} & 0.17 & $\mathrm{Si}$ & $\mathrm{Si}$ & $1 / 32^{\prime}$ & 8.0 & 10.0 & 11.5 & 13.0 & 14.0 \\
\hline & 0.27 & $\mathrm{Si}$ & Muy buena & $>1 / 32$ “ & 6.5 & 9.0 & 10.0 & 11.5 & 12.5 \\
\hline & 0.37 & $\mathrm{Si}$ & $\mathrm{Si}$ & $>1 / 32$ “ & 12.0 & 14.7 & 16.0 & 17.0 & 18.0 \\
\hline & 0.50 & $\mathrm{Si}$ & $\mathrm{Si}$ & $>1 / 32$ “ & 12.5 & 14.0 & 16.0 & 18.0 & 21.0 \\
\hline \multirow{4}{*}{1.5} & 0.17 & $\mathrm{Si}$ & $\mathrm{Si}$ & $1 / 32^{\prime}$ & 13.4 & 15.0 & 17.0 & 19.3 & 22.4 \\
\hline & 0.27 & $\mathrm{Si}$ & $\mathrm{Si}$ & $>1 / 32$ “ & 19.0 & 22.0 & 25.0 & 27.0 & 29.4 \\
\hline & 0.37 & $\mathrm{Si}$ & $\mathrm{Si}$ & $>1 / 32$ “ & 7.5 & 10.0 & 11.3 & 12.3 & 13.3 \\
\hline & 0.50 & $\mathrm{Si}$ & $\mathrm{Si}$ & $>1 / 32$ " & 6.0 & 7.5 & 8.0 & 8.5 & 9.7 \\
\hline
\end{tabular}

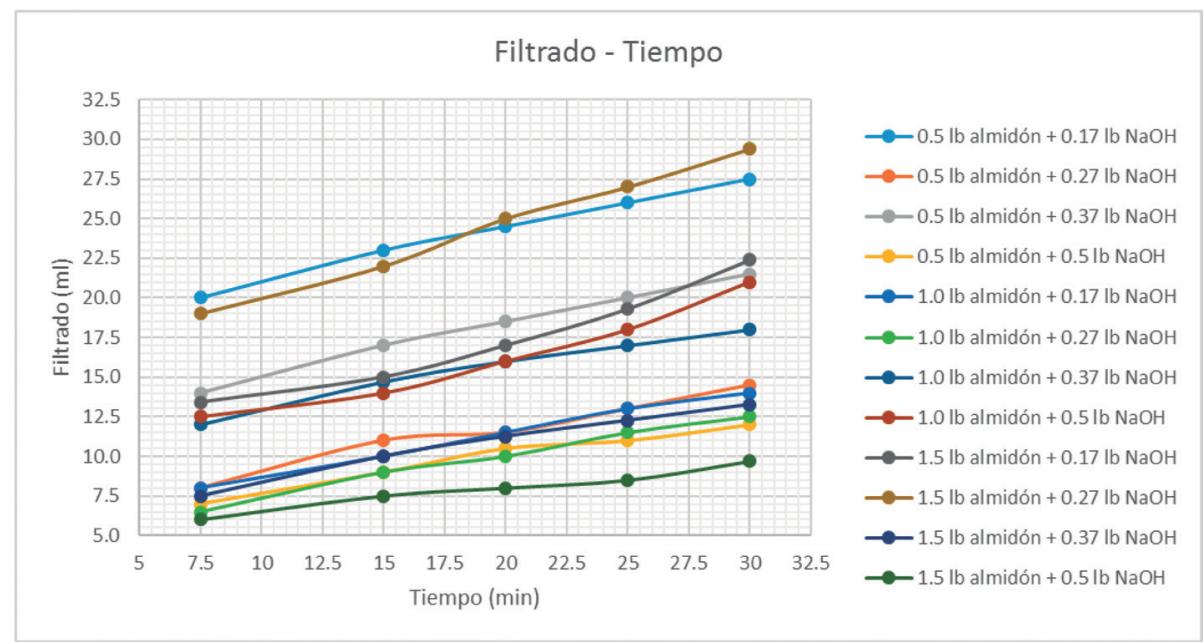

Figura $N^{\circ}$ 9. Pérdida Filtrado a través del tiempo.

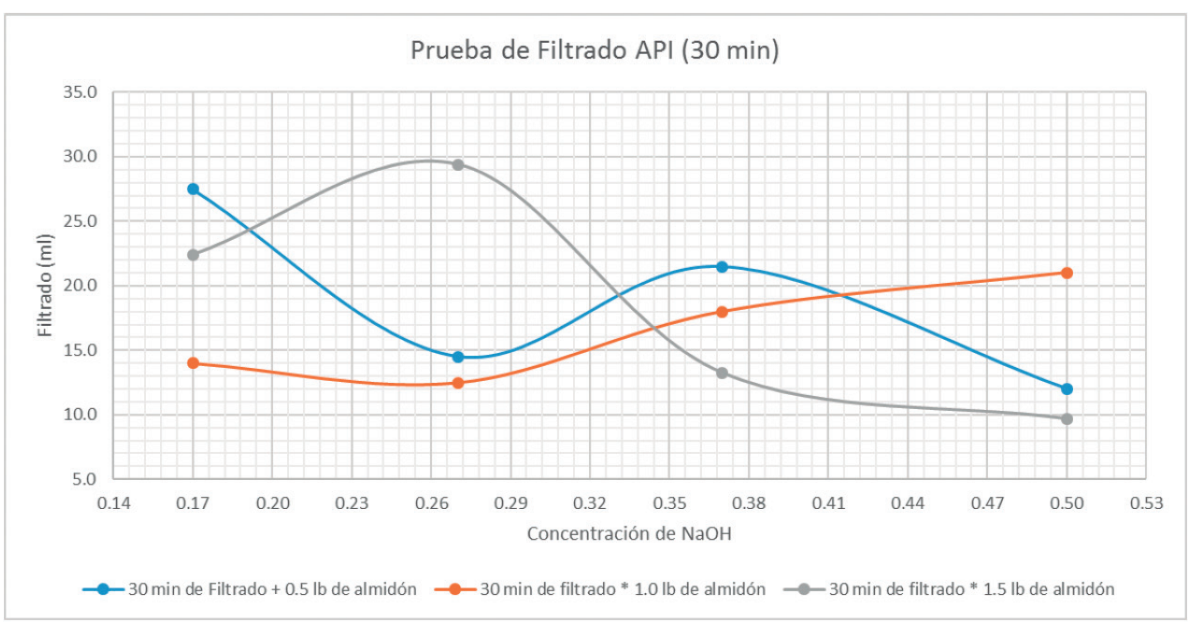

Figura $\mathbf{N}^{\circ}$ 10. Filtrado API (30 min). 
Luego de realizada las pruebas a las muestras nos indican que:

- A una concentración de $0.5 \mathrm{lb} / \mathrm{bbl}$ de almidón de papa se requieren altas concentraciones de $\mathrm{NaOH}$ para tener niveles óptimos de contenido de sólidos y agua.

- A una concentración de $1.0 \mathrm{lb} / \mathrm{bbl}$ de almidón de papa se requieren concentraciones intermedias de $\mathrm{NaOH}$ para tener niveles óptimos de contenido de sólidos y agua.

- A una concentración de $0.5 \mathrm{lb} / \mathrm{bbl}$ de almidón de papa se requieren bajas concentraciones de $\mathrm{NaOH}$ para tener niveles óptimos de contenido de sólidos y agua.

\begin{tabular}{|c|c|c|c|c|}
\hline \multicolumn{2}{|c|}{ CONTROL DE SOLIDOS } & ml de & $\begin{array}{c}\% \text { de } \\
\text { Líquidos }\end{array}$ & $\begin{array}{c}\% \text { de } \\
\text { Solidos }\end{array}$ \\
\hline $\begin{array}{c}\text { Almidón de } \\
\text { papa (lb) }\end{array}$ & $\begin{array}{c}\text { NaOH } \\
\text { (lb) }\end{array}$ & Destilado & & \\
\hline \multirow{4}{*}{0.5} & 0.17 & 9.60 & $96.0 \%$ & $4.0 \%$ \\
\cline { 2 - 5 } & 0.27 & 9.70 & $97.0 \%$ & $3.0 \%$ \\
\cline { 2 - 5 } & 0.37 & 9.70 & $97.0 \%$ & $3.0 \%$ \\
\cline { 2 - 5 } & 0.50 & 9.70 & $97.0 \%$ & $3.0 \%$ \\
\hline \multirow{4}{*}{1.0} & 0.17 & 9.30 & $93.0 \%$ & $7.0 \%$ \\
\cline { 2 - 5 } & 0.27 & 9.65 & $96.5 \%$ & $3.5 \%$ \\
\cline { 2 - 5 } & 0.37 & 9.80 & $98.0 \%$ & $2.0 \%$ \\
\cline { 2 - 5 } & 0.50 & 9.80 & $98.0 \%$ & $2.0 \%$ \\
\hline \multirow{4}{*}{1.5} & 0.17 & 9.70 & $97.0 \%$ & $3.0 \%$ \\
\cline { 2 - 5 } & 0.27 & 9.60 & $96.0 \%$ & $4.0 \%$ \\
\cline { 2 - 5 } & 0.37 & 9.90 & $99.0 \%$ & $1.0 \%$ \\
\cline { 2 - 5 } & 0.50 & 9.90 & $99.0 \%$ & $1.0 \%$ \\
\hline
\end{tabular}

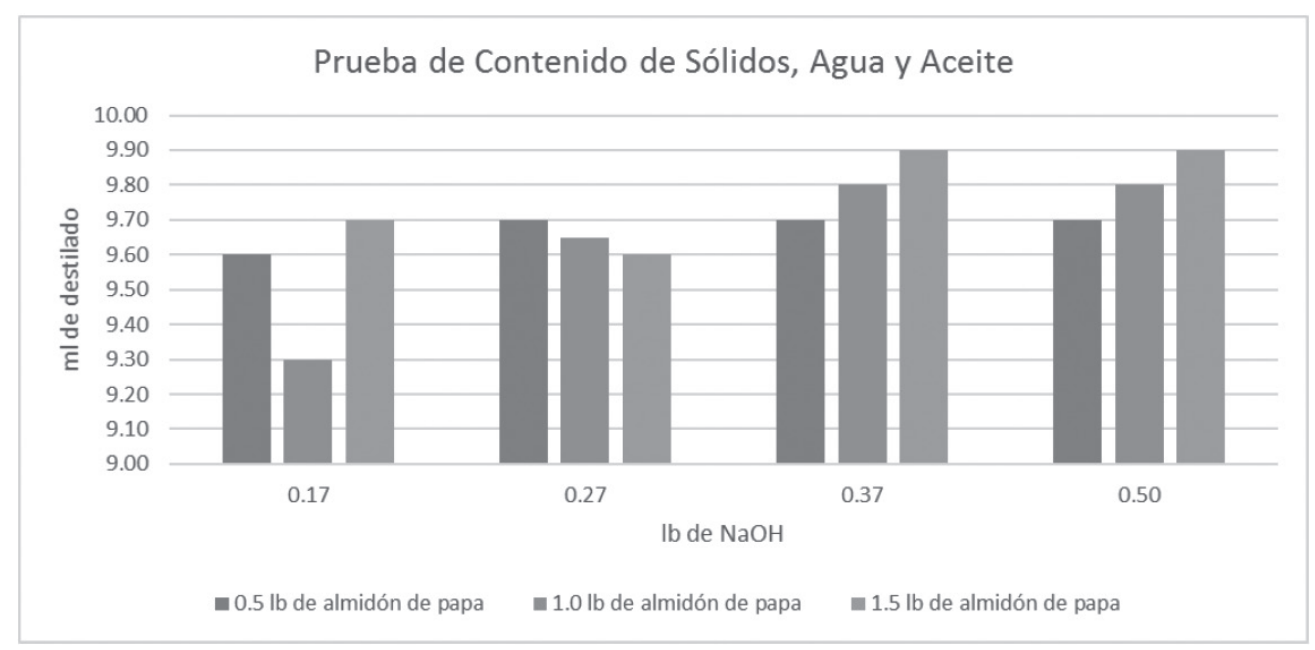

Figura $N^{\circ}$ 11. Prueba de contenido de sólidos, agua y aceite.

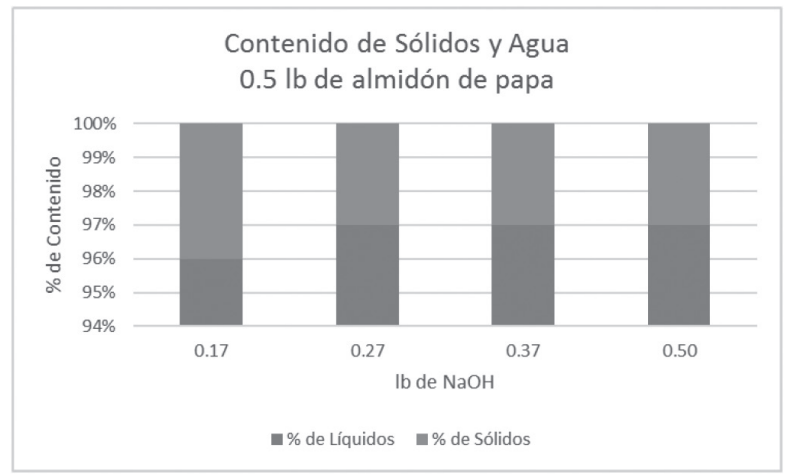

Figura $\mathbf{N}^{\circ}$ 12. Contenido de sólidos, agua y aceite para $0.5 \mathrm{lb}$ de almidón de papa.

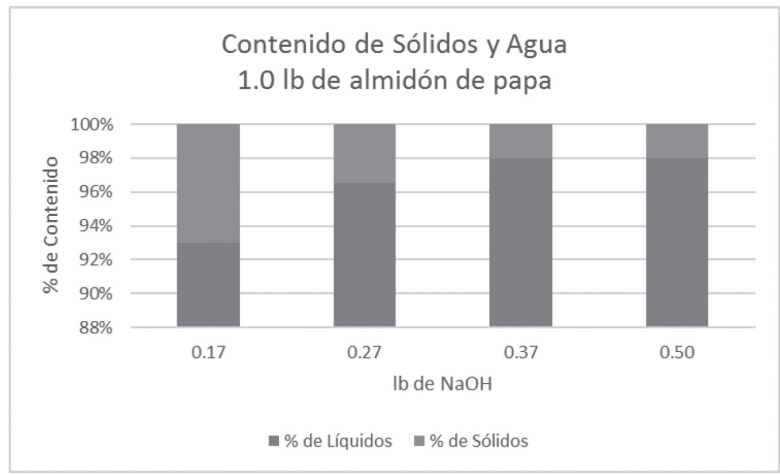

Figura $\mathbf{N}^{\circ}$ 13. Contenido de sólidos, agua y aceite para $1.0 \mathrm{lb}$ de almidón de papa. 


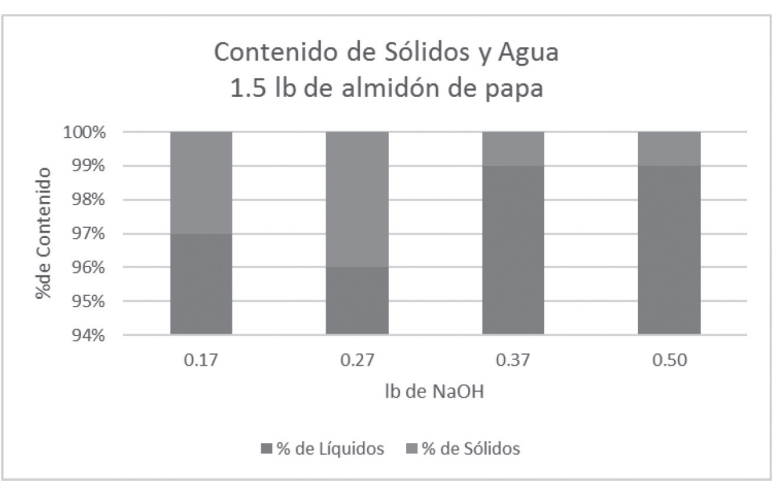

Figura $\mathbf{N}^{\circ}$ 14. Contenido de sólidos, agua y aceite para $1.5 \mathrm{lb}$ de almidón de papa

\section{Conclusiones}

- El almidón de papa actúa como mejorador de las propiedades viscosificantes de la bentonita.

- La densidad del lodo preparado con almidón de papa es $8.75 \mathrm{lb} /$ gal, siendo esta menor a uno que contiene PAC L, pero se encuentran dentro del rango óptimo establecido por el API.

- El almidón de papa a las concentraciones de $\mathrm{NaOH}$ experimentadas mantiene los niveles de $\mathrm{pH}$ en 10 , pero sufre activación térmica en concentraciones altas por el calor que genera la disolución de $\mathrm{NaOH}$, perdiendo así los niveles óptimos.

- Utilizando almidón de papa en reemplazo del PAC-L mejora el control de filtrado, la viscosidad y las propiedades reológicas (Viscosidad plástica, Yield point, viscosidad aparente, Esfuerzo de gel).

- El almidón de papa es un sustituto económico y ecológico del PAC-L como agente controlador de filtrado, contribuyendo con la preservación del medio ambiente, reducción de costos operacionales e industrialización.

\section{Recomendaciones}

- Realizar pruebas de rendimiento adicionales incrementando la relación de Almidón de Papa respecto a la Bentonita en $10 \mathrm{lb} / \mathrm{bbl}$.

- Evaluar la viscosidad y las propiedades reológicas del Almidón de Papa previa activación térmica.

\section{Agradecimientos}

Un agradecimiento especial a los ingenieros Alexander Beltrán, Erik Montes Paez y Mario Torres de la
Universidad Industrial de Santander por guiar, respaldar y contribuir en nuestras ideas. Al evaluador del articulo por las sugerencias y comentarios para mejorar este artículo.

A la escuela de Ingeniería de Petróleos de la Universidad Industrial de Santander, por brindarnos un espacio propicio para el aprendizaje y realización de las pruebas para la presente investigación.

\section{Referencias}

1.American Petroleum Insititute. (2017). Recommended Practice for field Testing Water-based Drilling Fluids (Fifth ed.). Washington DC, United States of America: API Publishing Services.

2. AMOCO Production Company. (1998). Drilling Fluids Manual (Sexta ed.). Illinios, Chicago, EEUU.

3. Ariza Leon, E., Castro Cely, A. L., \& Gómez Cañon, J. D. (2013, Junio). Factibilidad del uso del almidón de achira como agente controlador de fltrado en lodos de perforación base agua. ION, 63-71.

4. Ayala , F., Gómez, J., \& León, E. (2011). Estudio de factibilidad del uso de biodiesel como fase continua en lodos de perforación de emulsión inversa. Fuentes: El reventón energético, 9(1).

5. Ayala, D., Benítez, A., \& Valencia, R. (2017). Optimización de la Tasa de Penetración mediante el análisis de las vibraciones al perforar, caso de estudio Ecuador. Fuentes: El reventón energético, 15(1), 27-40.

6. Ayala, D., Torres, H., Valencia, R., \& Loaiza, M. (2016). Impacto del Tiempo no Productivo en operaciones de perforación y análisis de los datos mediante la 'prueba de Chicuadrado. Fuentes: El reventón energético, 14(2), 5-18.

7. Baker Hughes. (2006). Drilling Fluids Reference Manual. Houston, Texas, EEUU.

8. Bautista Cuadros, A. F., \& Flórez Barreto, E. D. (2011). Factibilidad del uso de Almidon de Yuca como aditivo en Lodos de Perforación. Tesis de Pregrado, Universidad Industrial de Santander, Escuela de Ingeniería de Petróleos, Bucaramanga.

9. Bemiller, J., \& Whistler, R. (2009). Starch: Chemistry and technology. (Third ed.). Burlington, MA, USA: Academic Press.

10. Campana, D., \& Tapia, R. (2017). Evaluación cualitativa de la limpieza de hoyo en pozos de alta inclinación-alto desplazamiento en la Cuenca Oriente. Fuentes: El reventón energético, 15(2), 49-56.

11. Cui, S. (2005). Food Carbohydrates: Chemistry, physical properties and applications (First ed.). 
LL, C, USA: Editorial CRC Press, Taylor \& Francias Group.

12. Direccion General de Seguimiento y Evaluación de Politicas - DGESEP. (2017). Boletín Estadístico de Producción Agrícola y Ganadera. Ministerio de Agricultura y Riego, Direccion General de Seguimiento y Evaluación de Politicas - DGESEP. Lima: Sistema Integrado de Estadistica Agraria.

13. Halliburton Baroid. (2017). PAC-L ${ }^{\mathrm{TM}}$. Houston, Texas, EE.UU.: Halliburton Baroid.

14. Kaur, A., Singh, N., Ezekiel, R., \& Guraya, H. S. (2007). Physicochemical, thermal and pasting properties of starches separated from different potato cultivars grown at different locations. Food Chemistry, 101(2), 643-651. doi:http:// dx.doi.org/10.1016/j.foodchem.2006.01.054.

15. Loaiza, M., Ayala, D., Torres, H., \& Ayala, S. (2018). Tiempo no productivo en pozos de dos secciones, caso de estudio Ecuador. Fuentes: El reventón energético, 16(1), 7-17.

16. Lyons, W., \& Plisga, G. (2004). Drilling and Well Completion. In W. C. Lyons, G. J. Plisga, \& W. C. Lyons (Ed.), Standard Handbook of Petroleum and Natural Gas Engineering (Second ed., Vol. I, pp. 513-1388). Houston, Texas, EE.UU.: Gulf Publishing Company. doi:https://doi.org/10.1016/B978-0750677851/50016-6

17. Medina, C., Martínez, J., León, E., \& Boada, W. (2013). Análisis reológico para predecir y mejorar el comportamiento hidráulico durante la perforación de un pozo. Fuentes: El reventón energético, 11(1).

18. MI -SWACO. (1998). MI - Drilling Engineering Manual (Primera ed.). Texas, Texas, USA.

19. MINAGRI. (2014, MAYO 5). Industrialización de la papa (en linea). Retrieved from MINAGRI: http://minagri.gob.pe/portal/objetivos/25sector-agrario/papa/214-industrializacion

20. Montes, A., Carreño , W., \& Guío, M. (2018). Aspectos de la perforación de pozos complejos en piedemonte en tiempos de crisis. Fuentes: El reventón energético, 16(1), 87-97.

21. Montgomery, D. C. (2004). DISEÑO Y ANALISIS
DE EXPERIMENTOS. Mexico DF: Editorial LIMUSA S.A.

22. Silva, D. A.V., Carillo, Z. C., \& Tarazona, D. M., (2018). Modelo matemático para determinar presión permitida de pérdidas de filtrado de lodo controladas. Fuentes: El reventón energético, 16(1), 47-55.

24. PDVSA CIED Petróleos de Venezuela, S.A. Centro Imternacional de Educación y Desarrollo. (2002). Fluidos de Perforación. Caracas, Caracas, Venezuela.

25. Peña Carrasco, E. F. (2017). Extracción y Caracterización Fisicoquímica y Funcional de Almidones de Cinco Variedades de Papas Nativas Procedentes de Ilave (Puno). Lima, Perú: Universidad Nacional Agraria La Molina.

26. Portilla, H., Suárez, D., \& Corzo, R. (2012). Metodología para la optimización de parámetros de perforación a partir de propiedades geomecánicas. Fuentes: El reventón energético, 10(2).

27. Rodriguez Bermudez, E. (2007). Reestructuración Teórico-Práctica de la guía de Laboratorio de Lodos y Cementos. Tesis de Grado, Universidad Industrial de Santander, Escuela de Ingeniería de Petróleos, Facultad de Ingenierías Fisicoquímicas, Bucaramanga, Colombia.

28. Sandhu, K. S., \& Singh, N. (2007). Some properties of corn starches II: Physicochemical, gelatinization, retrogradation, pasting and gel textural properties. Food Chemistry, 101(4), 1499-1507.

29. Schlumberger Limited. (2018, diciembre 3). Oilfield Glossary. Retrieved from Oilfield Glossary en Español: https://www.glossary.oilfield.slb. com/es/Terms/c/caustic_soda.aspx

30. Soto Izarra, R. D., \& Yantas Huaynate, P. E. (2012). Evaluación de la calidad del almidón obtenido de tres variedades de papa (Solanum tuberosum) cultivadas en la provincia de Jauja. Tesis de Grado, Universidad Nacional del Centro del Perú, Facultad de Ciencias Aplicadas, Escuela Académico Profesional de Ingeniería Agroindustrial, Tarma, Perú.

Recepción: 11 de junio de 2018

Aceptación: 06 de junio de 2019 